\title{
Correlation analysis between ultrasonography and mammography with other risk factors related to breast cancer
}

\author{
WENXIAO ZHANG $^{1 *}$, CUI XU ${ }^{1 *}$, RUI LI $^{1}$, GUANGHE CUI $^{1}$, MINMIN WANG $^{2}$ and MIN WANG ${ }^{1}$ \\ Departments of ${ }^{1}$ Ultrasound Medicine and ${ }^{2}$ Health Management, \\ Binzhou Medical University Hospital, Binzhou, Shandong 256600, P.R. China
}

Received November 26, 2018; Accepted April 4, 2019

DOI: $10.3892 /$ ol.2019.10246

\begin{abstract}
Value and correlation analysis between ultrasound and mammography in the diagnosis and other risk factors related to breast cancer were explored. A total of 1,473 patients who underwent breast color ultrasonography and molybdenum target X-ray examination in Binzhou Medical University Hospital from March 2017 to August 2018 were collected, and the patient's ultrasound and mammography results were compared, also the pathological biopsy was used as the reference golden criteria to calculate the value of both test methods and the value of combined diagnosis in breast cancer. The risk factors associated with breast cancer were analyzed. Among the 1,473 patients, 387 breast cancer patients were detected by ultrasonography, 351 by mammography and 339 cases by combined diagnosis. A total of 314 cases were diagnosed as breast cancer after pathological biopsy. However, there were significant differences in tumor size, stages, and BI-RADS grades $(\mathrm{P}<0.05)$. There was no significant difference in the diagnostic efficacy between ultrasonography and mammography $(\mathrm{P}>0.05)$, however, the diagnostic efficacy of ultrasonography combined with mammography was significantly better than the two single tests $(\mathrm{P}<0.05)$. After logistic regression analysis, there was no significant correlation between residence address, height, blood type, ethnicity, or education with breast cancer $(\mathrm{P}>0.05)$. However, age, fertility status, and BMI were all risk factors related to breast cancer $(\mathrm{OR}>1 ; \mathrm{P}<0.05)$. In conclusion, ultrasonography combined with mammography can effectively improve the early diagnosis rate of breast cancer, however, the patient's age, birth status, and BMI may affect the results of ultrasonography and mammography. In clinical practice, it is necessary to
\end{abstract}

Correspondence to: Dr Guanghe Cui, Department of Ultrasound Medicine, Binzhou Medical University Hospital, 661 Huanghe 2 Road, Binzhou, Shandong 256600, P.R. China E-mail: cq32t6@163.com

${ }^{*}$ Contributed equally

Key words: breast cancer, ultrasonography, mammography, influencing factors determine the imaging results in combination with the actual situation of the patients to improve the diagnosis rate of breast cancer.

\section{Introduction}

Breast cancer is the most common malignant tumor in women, its incidence rates have remained high for many years (1). According to statistics, there are more than 1 million cases of breast cancer diagnosed each year globally, and approximately 410,000 of them died from breast cancer (2). The incidence rate of breast cancer in some countries with dense populations and large population bases (such as China and India) were significantly higher than that of other countries (3). Also in recent years, there have been reports showing that the incidence has an increasingly obvious trend in the young (4,5). Orthotopic breast cancer does not have a high death rate, but once cancer cells metastasize, free cancer cells can be transferred anywhere through blood circulation and lymph circulation, then the threat of breast cancer is greatly increased (6). Therefore, the clinical practice of breast cancer advocates 'early detection, early treatment', during the time when in situ cancer has not yet spread, intervention therapy should be used to ensure the health of patients (7). Due to insignificant features in the early stage of breast cancer, most patients lack medical knowledge and do not pay attention to the onset of breast cancer, this leads to the causation of losing best time for treatment. Most patients have reached middle or late stage at the time of diagnosis, this is also one of the reasons for the rising incidence and mortality rates of breast cancer (8). Therefore, the key to reducing the mortality rate and improving the therapeutic effect of breast cancer is the method of how to diagnose it in an early stage of tumorigenesis. This is also a focused and difficult research in the current clinical practice. Ultrasound and molybdenum $\mathrm{X}$-ray target radiography are the most common imaging techniques used in clinical diagnosis of breast cancer. However, domestic research is currently limited to the diagnosis in the accuracy of breast cancer (9-11), the correlation between ultrasonography and mammography with other risk factors associated to breast cancer remains to be further investigated. By exploring the effects of breast cancer-related risk factors on acoustic and mammography, this may provide effective reference for breast cancer density assessment and standardization of dense breasts. It has a great significance for the early 
diagnosis of breast cancer. Therefore, this study analyzed the correlation among ultrasonography and mammography, and with other risk factors related to breast cancer, to provide reference and guidance for clinics.

\section{Materials and methods}

General information. A total of 1,473 patients who underwent breast color ultrasonography and mammography in Binzhou Medical University Hospital (Binzhou, China) from March 2017 to August 2018 were collected. The patients were aged 17-73 years, with an average age of $42.73 \pm 12.84$ years (Table I). This study was approved by the Ethics Committee of Binzhou Medical University Hospital, and each subject signed an informed consent form.

Inclusion and exclusion criteria. Inclusion criteria: i) patients met the clinical manifestations of breast cancer (12); ii) breast color ultrasonography and mammography X-ray examination were performed in Binzhou Medical University Hospital; iii) females; iv) patients willing to cooperate with the medical staff of Binzhou Medical University Hospital; v) consistent bilateral breast classification; and vi) with completed cases of medical records. Exclusion criteria: i) combine with other tumors; ii) severe organ failure; iii) abnormal liver and kidney function; iv) mental illness; v) a history of breast plastic surgery; vi) patients during pregnancy and lactation; vii) patients cannot take care of themselves; and viii) long-term bedridden.

Methods. Color ultrasonography was measured using the Acuson Sequoia 512 color ultrasound diagnostic instrument (Siemens AG, Munich, Germany). The patient was examined in a supine position and the upper limb was lifted, the chest and the bilateral lower jaw were fully exposed. Applying couplant around the center of the papillary, each quadrant of the breast to the bilateral axilla were detected successively. If a lump is found, the image is classified according to the visual observation, the number of mammary ducts, fat interstitial components and fibrous glandular tissue. Classification criteria were as previously described (13). Scno Advantage 2.1 digital mammography machine (GE Healthcare, Chicago, IL, USA) was used for molybdenum target X-ray examination, routine ingestion of unilateral or bilateral mammary axon and oblique position. When the development is unclear, partial pressure amplification photography is performed. Observing the type of breast [the classification criteria refer to the American Radiation Society breast impact report and statistics (14)] and lesion morphology. All the image results were reviewed by the 3 directors of the imaging department in Binzhou Medical University Hospital, and the patient's breast examination results were recorded.

Observation indices. In ultrasonography, breast cancer is determined when mammary gland is hard, inactive, and form orange-like change when it adheres to the skin, there is an incomplete boundary (crab-footed, jagged), with an uneven internal echo, and appears in the hypoechoic zone (15). In mammography, breast cancer is diagnosed when it is calcified, high or equal density tumors, with clear edges and invasive growth, along with visible radial line shadow, with distorted structure, and visible radial image and focal contraction or distorted at the substantial edge (16). When breast cancer is detected in both tests, it is determined by combined diagnosis. Reference golden criteria is pathological biopsy. To calculate the efficacy of the two examinations and combined diagnosis of breast cancer: Diagnostic sensitivity $(\%)=$ true positive/(true positive + false negative). Diagnostic specificity $(\%)=$ false positive/(false positive + true negative) . Diagnostic compliance rate $(\%)=$ (true positive + true negative)/(true positive + false positive + true negative + false negative). The correlation between both methods of detection and other risk factors related to breast cancer should also be analyzed.

Statistical analysis. Using SPSS 24.0 statistical software (Shanghai Yuchuang Network Technology Co., Ltd., Shanghai, China) to analyze and process the data, counting data is expressed in terms of (rate). Chi-square test was used for comparison between groups. Measurement data are expressed in the form of mean \pm standard deviation. The t-test was used for comparison between groups. Correlation analysis was performed using logistic regression analysis. $\mathrm{P}<0.05$ was considered to indicate a statistically significant difference.

\section{Results}

Diagnostic results. In the 1,473 patients, 387 breast cancer patients were detected by ultrasonography, 351 by mammography, 339 cases were detected by combined diagnosis and 314 were diagnosed as breast cancer after pathological biopsy. Also in the remaining 1,159 patients, 482 were diagnosed as breast adenosis, 294 as breast cysts, 257 as breast tuberculosis and 126 as mammary duct dilatation (data not shown).

Imaging features. The main expressions of breast cancer patients detected by utrasound examination are as follows: i) unclear boundaries and irregular shape, appearance more burr- and crab-like; ii) increased anteroposterior diameter of lesion, thickness/length $>1$; iii) attenuated rear echo, with more myeloid cells, loosen organizational structure and no echo enhancement after the lesion; iv) visible microcalcification in the lesion, a size of 100-500 $\mu \mathrm{m}$ and silent shadow behind; and v) rich blood flow signal in lesions, frequent high-speed and high-resistance blood flow. The main expressions of mammography are: i) irregular boundaries, high density nodular shadows with uneven density; and ii) burred edges, visible small clusters and gravel-like calcification (Figs. 1-3).

Ultrasound and mammography results. There were no significant differences in the results of tumor examination, tumor density, and lymph node metastasis between the methods $(\mathrm{P}>0.05)$. However, there were significant differences in tumor size, tumor stages, and BI-RAD grades $(\mathrm{P}<0.05$; Table II).

Diagnostic efficacy assessment. Taking pathological biopsy as the golden criteria, according to calculations, the sensitivity level of breast cancer detected by ultrasonography was $80.57 \%$, 
Table I. Clinical data of patients.

\begin{tabular}{lr}
\hline Factors & {$[\mathrm{n}(\%)]$} \\
\hline Age (years) & \\
$<42$ & $416(28.24)$ \\
$\geq 42$ & $1,057(71.76)$ \\
Height (cm) & \\
$<150$ & $515(34.96)$ \\
$\geq 150$ & $958(65.04)$ \\
Ethnicity & \\
Han & $1,321(89.68)$ \\
Others & $152(10.32)$ \\
Level of education & \\
$>$ high school & $876(59.47)$ \\
$<$ high school & $597(597)$ \\
Residence location & \\
Urban & $992(67.35)$ \\
Rural & $481(32.65)$ \\
BMI & \\
$<21$ & $531(22.47)$ \\
$\geq 21$ & $369(34.35)$ \\
Fertility status & \\
Fertilized & \\
Unfertilized & \\
Blood type & \\
A & \\
B & \\
O & \\
AB & \\
\hline
\end{tabular}

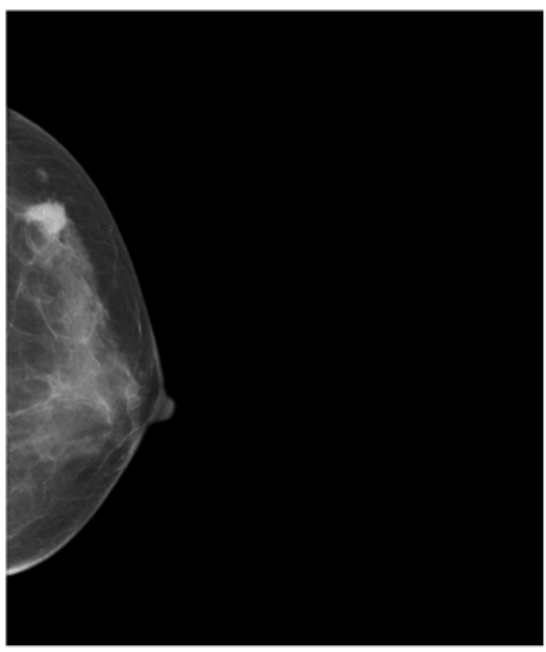

Figure 1. Irregular nodular high-density shadow on the upper outer quadrant of the left breast, there are long and short burrs around, and multiple fine-grained/small polymorphic calcifications are visible inside. Similar to BI-RADS Category 5, considered as breast cancer.

the specificity was $88.43 \%$ and the diagnostic compliance rate was $93.55 \%$. The sensitivity level of breast cancer detected

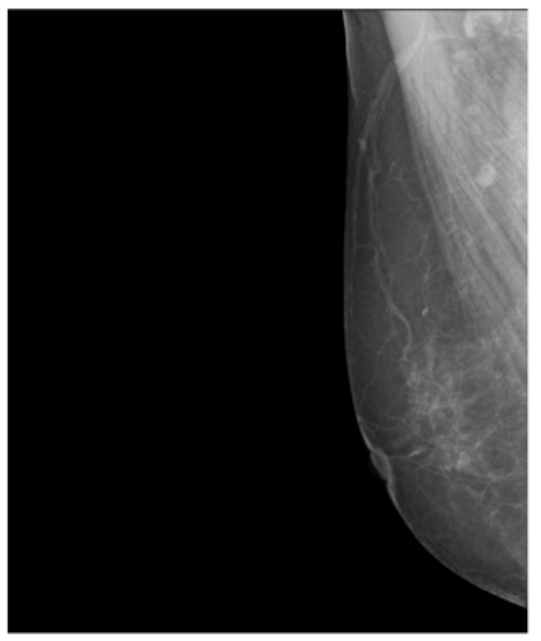

Figure 2. Nodular high-density foci on the upper left quadrant, irregular lesion boundary, with a rough local margin (invasive change, obvious CC position). Similar to BI-RADS Category 5 , considered as breast cancer.

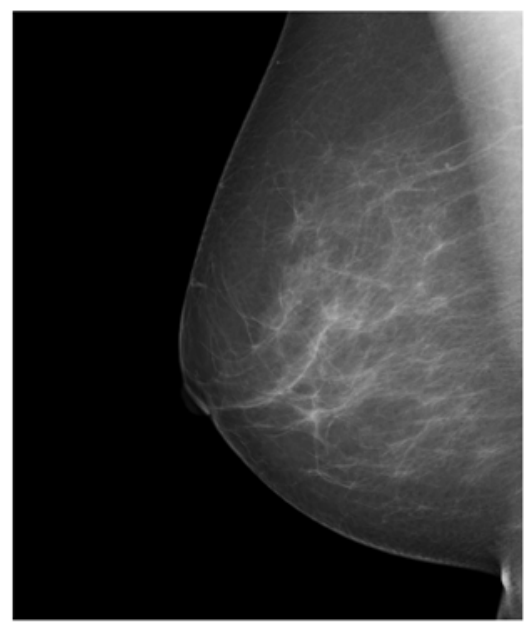

Figure 3. High-density mass on the upper left quadrant, with a clear lesion boundary and irregular boundary of posterior margin, invasive change. Also 'sharp-looking' protrusions and burrs. Similar to BI-RADS Category 5, considered as breast cancer.

by mammography was $83.35 \%$, the specificity was $92.83 \%$ and the diagnostic compliance rate was $91.24 \%$. The sensitivity level of breast cancer detected by combined diagnosis was $97.45 \%$, the specificity was $97.15 \%$ and the diagnostic compliance rate was $97.22 \%$. There was no significant difference in the diagnostic efficacy between ultrasonography and mammography $(\mathrm{P}>0.05)$. However, the diagnostic efficacy of ultrasonography combined with mammography was significantly better than the other two single tests $(\mathrm{P}<0.05$; Tables III-V $)$.

Analysis of risk factors in mammary gland. After logistic regression analysis, there was no significant correlation between residence location, height, blood type, ethnicity or level of education with breast cancer $(\mathrm{P}>0.05)$. However, age, fertility, and BMI were closely related to breast cancer $(\mathrm{P}<0.05)$. Also all constituted risk factors for breast cancer $(\mathrm{OR}>1$; Table VI). 
Table II. Comparison of ultrasound and mammography results [n (\%)].

\begin{tabular}{|c|c|c|c|c|}
\hline & Ultrasonography (n=387) & Mammorgraphy $(\mathrm{n}=351)$ & $\chi^{2}$ & P-value \\
\hline Types of tumor & & & 3.130 & 0.077 \\
\hline Invasive ductal carcinoma & $70(18.09)$ & $82(23.36)$ & & \\
\hline Carcinoma in situ & $317(81.91)$ & $269(76.64)$ & & \\
\hline Tumor sizes $(\mathrm{cm})$ & & & 8.464 & 0.015 \\
\hline$<1.0$ & $167(43.15)$ & $119(33.90)$ & & \\
\hline $1.1-5.0$ & $218(56.33)$ & $226(64.39)$ & & \\
\hline$>5.0$ & $2(0.52)$ & $6(1.71)$ & & \\
\hline Tumor stages & & & 15.412 & $<0.001$ \\
\hline Stage I-II & $315(81.40)$ & $242(68.95)$ & & \\
\hline Stage III-IV & $72(18.60)$ & $109(31.05)$ & & \\
\hline BI-RADS grades & & & 119.823 & $<0.001$ \\
\hline 3 & $7(1.81)$ & $1(0.28)$ & & \\
\hline 4 & $282(72.87)$ & $121(34.47)$ & & \\
\hline 5 & $98(25.32)$ & $229(65.24)$ & & \\
\hline Tumor density & & & 3.256 & 0.071 \\
\hline Low density & $47(12.14)$ & $59(16.81)$ & & \\
\hline High density & $340(87.86)$ & $292(83.19)$ & & \\
\hline Lymph node metastasis & & & 0.538 & 0.463 \\
\hline Exist & $32(8.27)$ & $24(6.84)$ & & \\
\hline Non-existent & $355(91.73)$ & $327(93.16)$ & & \\
\hline
\end{tabular}

Table III. Ultrasound diagnosis of breast cancer results.

\begin{tabular}{lcrr}
\hline Diagnostic method & Biopsy (+) & Biopsy (-) & \\
\hline Ultrasound (+) & 253 & 134 & 387 \\
Ultrasound (-) & 61 & 1,025 & 1,086 \\
& 314 & 1,159 & \\
\hline
\end{tabular}

Table IV. Mammography diagnosis of breast cancer results.

\begin{tabular}{lccr}
\hline Type & Biopsy (+) & Biopsy (-) & \\
\hline Mammography (+) & 268 & 83 & 351 \\
Mammography (-) & 46 & 1,076 & 1,122 \\
& 314 & 1,159 & \\
\hline
\end{tabular}

\section{Discussion}

Breast cancer, as the most common malignant tumor in women globally, its incidence and mortality rates are showing an upward trend (17). In order to improve the diagnosis and treatment of breast cancer; clinically, we are currently working on the diagnosis and treatment of pathogenesis of breast cancer from various angles. However, still no significant breakthrough has yet been made. For the pathogenesis of breast cancer, there is still a big controversy in the clinics. Poortmans et al (18) considered that the incidence of breast cancer is mainly due to
Table V. Combined diagnosis of breast cancer results.

\begin{tabular}{lrrr}
\hline Diagnostic type & Biopsy (+) & Biopsy (-) & \\
\hline Combined diagnosis (+) & 306 & 33 & 339 \\
Combined diagnosis (-) & 8 & 1,126 & 1,134 \\
& 314 & 1,159 & \\
\hline
\end{tabular}

Table VI. Logistic regression analysis of other risk factors related to breast cancer.

\begin{tabular}{lccc}
\hline Factors & OR & $95 \% \mathrm{CI}$ & P-value \\
\hline Age & 1.51 & $1.04-2.18$ & 0.028 \\
Height & 1.11 & $0.72-1.70$ & 0.648 \\
BMI & 1.72 & $1.05-2.83$ & 0.031 \\
Residence location & 1.07 & $0.71-1.62$ & 0.745 \\
Blood type & 0.59 & $0.07-5.06$ & 0.632 \\
Ethnicity & 0.76 & $0.37-1.59$ & 0.471 \\
Level of education & 0.88 & $0.61-1.29$ & 0.527 \\
Fertility & 1.64 & $1.09-2.47$ & 0.017 \\
\hline
\end{tabular}

the role of genetic factors, however, Belani et al (19) showed that the incidence of breast cancer is closely related to cancer stem cells. Currently, there is no accurate and reliable study for the exact cause of breast cancer, the main diagnostic methods in clinics are still carried out by imaging. As the disease 
progresses breast cancer is more difficult to be effectively diagnosed by imaging alone (20), therefore summarizing the risk factors of breast cancer has become a very important research topic for the impact of imaging examination. Traditional ultrasonography and mammography are simple, convenient, non-invasive, and have a long history of diagnosis. This is an advantage of breast cancer diagnosis, but there is a serious controversy on the determination of benign masses and cystic hyperplasia of the breast. We studied the diagnostic efficacy of ultrasonography and mammography for breast cancer, and analyzed the impact of breast cancer risk factors. This is to provide accurate guidance for clinical diagnosis and treatment of breast cancer.

The results of the experiments show, by comparing the patients with BI-RADS grades 4 and 5, that the effect of using mammography was better. The main reason is that it has a higher probability of calcification in breast cancer patients at this stage, and it has an excellent imaging effect on calcification when the mammography is performed (21), therefore, the diagnostic effect is better than that of ultrasonography. Also it suggested the necessity to perform a pathological biopsy as soon as possible to confirm the diagnosis when the suspected breast cancer is diagnosed as a BI-RADS grades 4 and 5 in the mammography. For the invasive ductal carcinoma and patients with grades II and III breast cancer, the diagnostic rate of applying ultrasongraphy was significantly higher than that of the mammography. It is suggested that it can be further confirmed by ultrasound examination for clinical early occult breast cancer that failed to pass mammography. The application of ultrasongraphy and mammography has good sensitivity and specificity for breast cancer. However, the combined diagnosis of both methods is even more effective in the diagnosis of breast cancer. It is suggested that the clinical diagnosis of breast cancer can be improved by the combined examination of ultrasonography and mammography. This is also consistent with the research results of Zhang et al (22), and further supports the results of our study. The molybdenum target $\mathrm{X}$-ray is superior to ultrasonography in the diagnosis of malignant breast tumors. However, ultrasonography is more accurate for mammary cystic hyperplasia and benign mass than molybdenum target X-ray (23). Most breast cancer patients have low echo signals in breast ductal fibrosis, so occult breast cancer with dense breast tissue can be detected by ultrasonography and this is not available for molybdenum target $\mathrm{X}$ cues (24). By combining both methods, it can make up for the shortcomings and achieve the best diagnosis results.

Logistic regression analysis found that age, fertility, and BMI were risk factors for breast cancer. Analysis of its effects with ultrasonography and mammography may be based on: i) with the increasing age, the ducts of the breast begin to degenerate and become thinner. This also caused the mammary gland to be slowly connected and covered with adipose tissue (25). At this time, imaging examination may result in the inability to distinguish breast end catheters that have shrunk or disappeared. Also the addition of other fibrous tissues (such as connective, fat and lobular) will affect the results of the image. ii) The mammary gland can directly affect the secretion of sex hormones by acting as a female sexual organ (26). The differentiation of mammary gland cells in pregnant and lactating women is more mature and has a longer survival period (26). Epithelial and acinar hyperplasia of the mammary duct may result in a significant increase in intra-lobular ducts within the breast, which is not-conducive for imaging examination. Moreover, the decrease in the level of sex hormones reduces the ability of proliferation in cells. Atrophic epithelial cells and fat may replace the original metabolic cells in the mammary gland, this is also one of the causes of errors that may affect the image results. iii) Excessive body fat can cause insulin resistance, and stimulate steroid-induced atypical proliferation of mammary epithelial cells (27). A large reduction in the composition of the glandular gland tissue causes the original mammary gland structure to be destroyed by adipose tissues and further cause deviations in image results.

This study mainly investigated the value of ultrasonography and mammography in the diagnosis of breast cancer, explored the correlation between ultrasonography, mammography and other risk factors related to breast cancer. However, there are still shortcomings due to limited experimental conditions. The study population is relatively similar, and there may be differences in different ethnic groups. Also the number of samples is small. Thus, further investigations are needed for confirmation.

In summary, the use of ultrasonography combined with mammography can effectively improve the early diagnosis rate of breast cancer. The patient's age, fertility status, and BMI may affect the results of ultrasonogrpahy and mammography. In clinical practice, it is necessary to determine the imaging results in combination with the actual situation of the patients in order to improve the diagnosis rate of breast cancer.

\section{Acknowledgements}

Not applicable.

\section{Funding}

No funding was received.

\section{Availability of data and materials}

The datasets used and/or analyzed during the present study are available from the corresponding author on reasonable request.

\section{Authors' contributions}

WZ and RL were responsible for color ultrasonography analysis, GC and MinminW recorded and analyzed general data of patients. CX and MinW helped with analysis of observation indices. The final version was read and adopted by all the authors.

\section{Ethics approval and consent to participate}

The study was approved by the Ethics Committee of Binzhou Medical University Hospital (Binzhou, China). Signed informed consents were obtained from the patients or the guardians. 


\section{Patient consent for publication}

Not applicable.

\section{Competing interests}

The authors declare that they have no competing interests.

\section{References}

1. DeSantis CE, Fedewa SA, Goding Sauer A, Kramer JL, Smith RA and Jemal A: Breast cancer statistics, 2015: Convergence of incidence rates between black and white women. CA Cancer J Clin 66: 31-42, 2016.

2. Coughlin SS and Ekwueme DU: Breast cancer as a global health concern. Cancer Epidemiol 33: 315-318, 2009.

3. Li T, Mello-Thoms C and Brennan PC: Descriptive epidemiology of breast cancer in China: incidence, mortality, survival and prevalence. Breast Cancer Res Treat 159: 395-406, 2016.

4. Ghoncheh M, Pournamdar Z and Salehiniya H: Incidence and mortality and epidemiology of breast cancer in the world. Asian Pac J Cancer Prev 17 (S3): 43-46, 2016.

5. Han W and Kang SY; Korean Breast Cancer Society: Relationship between age at diagnosis and outcome of premenopausal breast cancer: age less than 35 years is a reasonable cut-off for defining young age-onset breast cancer. Breast Cancer Res Treat 119: 193-200, 2010.

6. Ercan C, van Diest PJ and Vooijs M: Mammary development and breast cancer: the role of stem cells. Curr Mol Med 11: 270-285, 2011.

7. Collignon J, Lousberg L, Schroeder H and Jerusalem G: Triple-negative breast cancer: treatment challenges and solutions Breast Cancer (Dove Med Press) 8: 93-107, 2016.

8. Miller KD, Siegel RL, Lin CC, Mariotto AB, Kramer JL, Rowland JH, Stein KD, Alteri R and Jemal A: Cancer treatment and survivorship statistics, 2016. CA Cancer J Clin 66: 271-289, 2016.

9. Welch HG, Prorok PC, O'Malley AJ and Kramer BS: Breast-cancer tumor size, overdiagnosis, and mammography screening effectiveness. N Engl J Med 375: 1438-1447, 2016.

10. Ohuchi N, Suzuki A, Sobue T, Kawai M, Yamamoto S, Zheng YF, Shiono YN, Saito H, Kuriyama S, Tohno E, et al; J-START investigator groups: Sensitivity and specificity of mammography and adjunctive ultrasonography to screen for breast cancer in the Japan Strategic Anti-cancer Randomized Trial (J-START): a randomised controlled trial. Lancet 387: 341-348, 2016.

11. McDonald ES, Oustimov A, Weinstein SP, Synnestvedt MB, Schnall $\mathrm{M}$ and Conant EF: Effectiveness of digital breast tomosynthesis compared with digital mammography: outcomes analysis from 3 years of breast cancer screening. JAMA Oncol 2: 737-743, 2016.

12. Veronesi U, Boyle P, Goldhirsch A, Orecchia R and Viale G: Breast cancer. Lancet 365: 1727-1741, 2005.

13. Tadayyon H, Sadeghi-Naini A, Wirtzfeld L, Wright FC and Czarnota G: Quantitative ultrasound characterization of locally advanced breast cancer by estimation of its scatterer properties. Med Phys 41: 012903, 2014.

14. Pfarl G and Helbich TH: Breast Imaging Reporting and Data System (BI-RADS)-German version. Rofo 174: 921-926, 2002 (In German)
15. Giuliano V and Giuliano C: Improved breast cancer detection in asymptomatic women using 3D-automated breast ultrasound in mammographically dense breasts. Clin Imaging 37: 480-486, 2013.

16. Olsen $\mathrm{O}$ and Gøtzsche PC: Screening for breast cancer with mammography. Cochrane Database Syst Rev 4: CD001877, 2001.

17. Oh CM, Won YJ, Jung KW, Kong HJ, Cho H, Lee JK, Lee DH and Lee KH; Community of Population-Based Regional Cancer Registries: Cancer statistics in Korea: incidence, mortality, survival, and prevalence in 2013. Cancer Res Treat 48: 436-450, 2016.

18. Poortmans PM, Collette S, Kirkove C, Van Limbergen E, Budach V, Struikmans H, Collette L, Fourquet A, Maingon P, Valli M, et al; EORTC Radiation Oncology and Breast Cancer Groups: Internal mammary and medial mupraclavicular irradiation in breast cancer. N Engl J Med 373: 317-327, 2015.

19. Belani CP, Schreeder MT, Steis RG, Guidice RA, Marsland TA, Butler EH and Ramalingam SS: Cetuximab in combination with carboplatin and docetaxel for patients with metastatic or advanced-stage nonsmall cell lung cancer: a multicenter phase 2 study. Cancer 113: 2512-2517, 2008.

20. Bianchini G, Balko JM, Mayer IA, Sanders ME and Gianni L: Triple-negative breast cancer: challenges and opportunities of a heterogeneous disease. Nat Rev Clin Oncol 13: 674-690, 2016.

21. Dobrosavljević A, Rakić S, Nikoli B, Raznatović SJ, Dikić SD, Milosević Z, Jurisić A and Skrobić M: Diagnostic value of breast ultrasound in mammography BI-RADS 0 and clinically indeterminate or suspicious of malignancy breast lesions. Vojnosanit Pregl 73: 239-245, 2016.

22. Zhang H, Tan H, Gao J, Wei Y, Yu Z and Zhou Y: The use of sequential X-ray, CT and MRI in the preoperative evaluation of breast-conserving surgery. Exp Ther Med 12: 1275-1278, 2016.

23. Tsigginou A, Gkali C, Chalazonitis A, Feida E, Vlachos DE, Zagouri F, Rellias I and Dimitrakakis C: Adding the power of iodinated contrast media to the credibility of mammography in breast cancer diagnosis. Br J Radiol 89: 20160397, 2016.

24. Bae S, Yoon JH, Moon HJ, Kim MJ and Kim EK: Breast microcalcifications: diagnostic outcomes according to image-guided biopsy method. Korean J Radiol 16: 996-1005, 2015.

25. Chen HL, Zhou MQ, Tian W, Meng KX and He HF: Effect of age on breast cancer patient prognoses: a population-based study using the SEER 18 database. PLoS One 11: e0165409, 2016.

26. Mullooly M, Yang HP, Falk RT, Nyante SJ, Cora R, Pfeiffer RM, Radisky DC, Visscher DW, Hartmann LC, Carter JM, et al: Relationship between crown-like structures and sex-steroid hormones in breast adipose tissue and serum among postmenopausal breast cancer patients. Breast Cancer Res 19: 8, 2017.

27. Jeon SY, Hwang KA and Choi KC: Effect of steroid hormones, estrogen and progesterone, on epithelial mesenchymal transition in ovarian cancer development. J Steroid Biochem Mol Biol 158: $1-8,2016$

This work is licensed under a Creative Commons Attribution-NonCommercial-NoDerivatives 4.0 International (CC BY-NC-ND 4.0) License. 